\title{
Spontaneous Breathing during Partial Liquid Ventilation in Animals with Meconium Aspiration
}

\author{
HELMUT D. HUMMLER, ULRICH THOME, ANDREAS SCHULZE, ROLF SCHNABEL, \\ FRANK POHLANDT, AND AXEL R. FRANK \\ Division of Neonatology and Pediatric Critical Care, Children's Hospital, University of Ulm, 89070 Ulm, \\ Germany [H.D.H., U.T., F.P., A.R.F.]; Division of Neonatology, Department of Obstetrics and \\ Gynecology, Grosshadern, Ludwig-Maximilian-University, 81377 Munich, Germany [A.S.]; and Institute \\ for Pathology, 45659 Recklinghausen, Germany [R.S.]
}

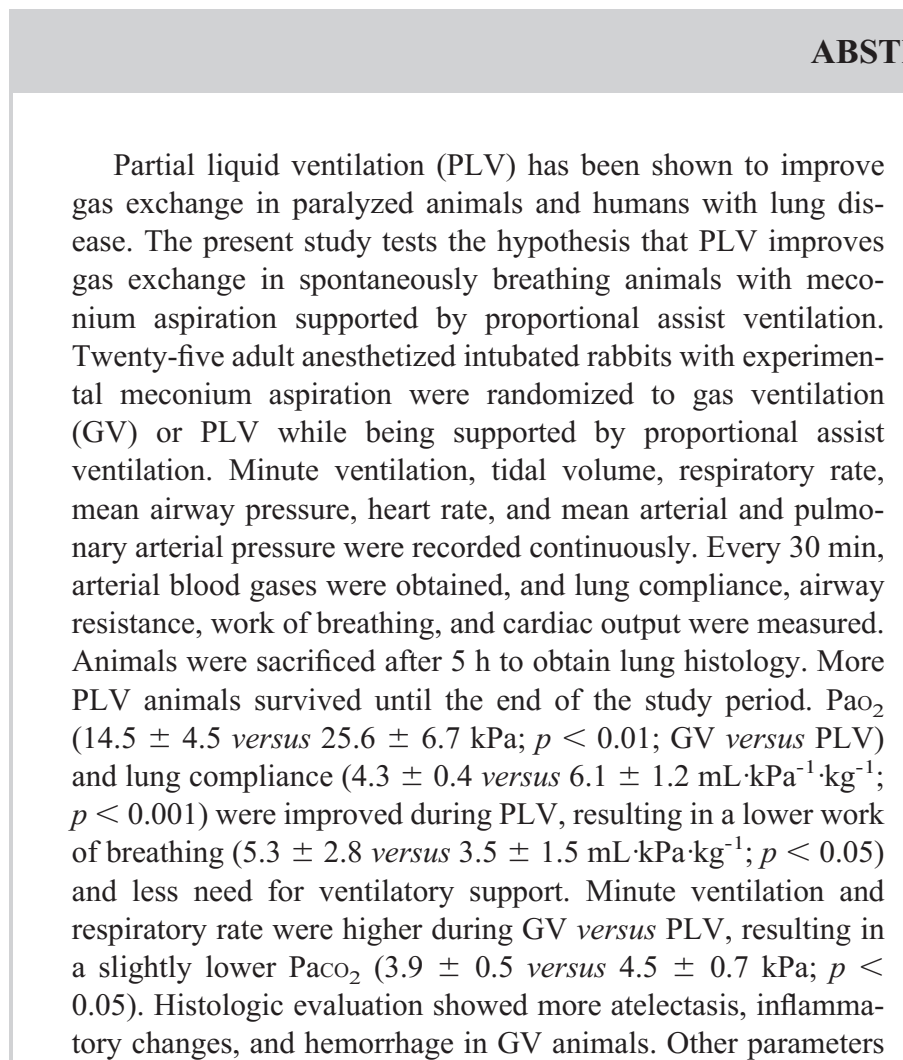

Previous studies have shown that PLV can improve gas exchange in animals and human neonates with severe lung disease (1-4). In most studies, PLV has been tested in pharmacologically paralyzed subjects and not during preserved spontaneous respiratory activity. However, there are potential

Received August 14, 2000; accepted December 8, 2000.

Correspondence and reprint requests: Helmut D. Hummler, M.D., Division of Neonatology and Pediatric Critical Care, Children's Hospital, University of Ulm, 89070 Ulm, Germany; e-mail: helmut.hummler@medizin.uni-ulm.de

Supported by a grant from the German Research Foundation (Deutsche Forschungsgemeinschaft; DFG HU 793/1-1).

Presented at the annual meeting of the Society of Pediatric Research, San Francisco, 1999. measured were similar. We conclude that PLV improves oxygenation, lung compliance, and survival and results in less lung injury in spontaneously breathing animals with meconium aspiration when supported by proportional assist ventilation. (Pediatr Res 49: 572-580, 2001)
b.w., body weight
Abbreviations
GV, gas ventilation
Ke, degree of elastic unloading
$\mathbf{K r}$, degree of resistive unloading
MAP, mean airway pressure
MA, meconium aspiration
PAV, proportional assist ventilation
Paw, airway pressure
Pe, esophageal pressure
PEEP, positive end-expiratory pressure
PFC, perfluorocarbon chemical
PLV, partial liquid ventilation
$\mathbf{R R}$, respiratory rate
$\mathrm{SaO}_{2}$, arterial oxygen saturation
$\mathrm{Svo}_{2}$, mixed venous oxygen saturation
$\dot{\mathbf{V}}$, minute ventilation
WOB, work of breathing

disadvantages of neuromuscular blockade in ventilated infants, such as atrophy of respiratory muscles (5), impaired pulmonary mechanics (6), and a lower functional residual capacity resulting in impaired oxygenation (7). On the other hand, it has been shown that preserved spontaneous breathing during mechanical ventilation may improve ventilation-perfusion match, cardiac output, and blood pressure (8). Furthermore, synchronizing ventilator breaths with the patient's own respiratory effort may increase the efficiency of mechanical ventilation (9), improve oxygenation (10), and may decrease the incidence of chronic lung disease in neonates (11). More recently, it has been shown that assisted modes of ventilation may be combined with PLV to support spontaneously breathing animals without lung dis- 
ease $(12,13)$ and with acute respiratory distress syndrome (ARDS) (14).

Respiratory mechanical unloading, also called PAV, is a recently developed mode of ventilatory support $(15,16)$ that adjusts the applied Paw within each respiratory cycle in proportion to the patient's effort. Thus, the depth, timing, and pressure profile of each ventilator breath is controlled by the patient $(15,16)$. Therefore, it may provide potential advantages, such as improved patient comfort, preservation of the subject's own reflex and control mechanisms, and lower peak and MAP to maintain ventilation (17).

In addition, combination of PLV and mechanical unloading may be useful to compensate for decreased lung compliance in subjects with lung disease and for increased resistance caused by the liquid within the airways during $\operatorname{PLV}(13,18,19)$. We have previously shown that stable ventilation and gas exchange may be achieved during PLV in spontaneously breathing animals supported by PAV in the absence of lung disease (13). The aim of this study was to test this mode of ventilation in spontaneously breathing animals with severe MA. We hypothesized that PLV improves oxygenation also in spontaneously breathing animals with severe MA supported by PAV compared with a control group not treated with PLV.

\section{METHODS}

The present study was approved by the Animal Care Committee of the government agencies of Baden-Württemberg.

Ventilator. A Stephanie infant ventilator (Stephan Medizintechnik $\mathrm{GmbH}$, Gackenbach, Germany) was used throughout the study. In addition to conventional ventilation, this ventilator provides resistive and elastic unloading. The device has been described in detail elsewhere $(15,17,20)$. It is a servocontrolled system that uses the flow signal obtained with a pneumotachograph (dead space, $0.6 \mathrm{~mL}$ ). After processing, the signal is used to control a rapid valve that determines the Paw at the endotracheal tube. The pressure applied per unit airflow determines the Kr. The pressure applied per unit of inspired volume determines the Ke. The delivered Paw is a weighted sum of the resistive and the elastic components at any point in time during a spontaneous breathing cycle: $\mathrm{Paw}=(\mathrm{Kr} \cdot \dot{\mathrm{V}})+$ $(\mathrm{Ke} \cdot \mathrm{Vt})+\mathrm{PEEP}$, where $\dot{\mathrm{V}}$ is flow, $\mathrm{Vt}$ is volume, and PEEP is the positive end-expiratory pressure.

Animal preparation. Twenty-five female adult New Zealand White rabbits with a b.w. of $2918 \pm 173 \mathrm{~g}$ (mean \pm SD) were given $0.2 \mathrm{mg} / \mathrm{kg}$ atropine and were anesthetized with $5-13$ $\mathrm{mg} / \mathrm{kg}$ b.w. ketamine and $0.5-1.3 \mathrm{mg} / \mathrm{kg}$ b.w. xylazine i.v. After supine positioning, the animals were intubated with a 3.5-mm inner-diameter cuffed endotracheal tube, and the cuff was inflated to prevent leaks. A rectal temperature probe (Siemens Sirecust 302, Erlangen, Germany) was placed, and a core temperature of $38-39.5^{\circ} \mathrm{C}$ was maintained using a heating blanket and an overhead warmer (Babytherm 8000, Draeger, Luebeck, Germany). Anesthesia was maintained by a continuous infusion of ketamine 65 (37-100) [median (range)] mg/kg b.w./h. The dose was adjusted to maintain anesthesia deep enough to prevent spontaneous movements other than respiration. During the surgical instrumentation, the animals were placed on volume-controlled synchronized intermittent positive pressure ventilation with the following settings: $\mathrm{FiO}_{2}, 0.4$; tidal volume, $10 \mathrm{~mL} / \mathrm{kg}$; PEEP, $0.4 \mathrm{kPa}\left(4 \mathrm{~cm} \mathrm{H}_{2} \mathrm{O}\right)$; inspiratory time, $0.4 \mathrm{~s} ; \mathrm{RR}, 30 / \mathrm{min}$. The rate was adjusted to maintain a $\mathrm{PaCO}_{2}$ within the target range $(4.7-6.7 \mathrm{kPa} ; 35-50 \mathrm{~mm} \mathrm{Hg})$. Dextrose $5 \%$ with $70 \mathrm{mmol} / \mathrm{L} \mathrm{Na}, 18 \mathrm{mmol} / \mathrm{L} \mathrm{K}$, and $1 \mathrm{U} / \mathrm{mL}$ heparin was administered at $5 \mathrm{~mL} / \mathrm{kg}$ b.w./h into a peripheral vein. A 3.5F arterial femoral line was inserted for continuous blood pressure monitoring and sampling for blood gas analyses. The line was continuously flushed with heparinized (1 $\mathrm{U} / \mathrm{mL}$ ) normal saline at a rate of $3 \mathrm{~mL} / \mathrm{h}$. A 4-lumen $4 \mathrm{~F}$ thermodilution catheter was introduced via the right jugular vein. Its tip was successfully placed into the pulmonary artery in 18 out of 25 animals to measure pulmonary artery pressure, central venous pressure, and cardiac output by thermodilution with a Sat-2 cardiac output monitor (Baxter, Santa Ana, CA, U.S.A.). Pulmonary arterial and central venous lines were continuously flushed with heparinized $(1 \mathrm{U} / \mathrm{mL})$ normal saline at a rate of $2 \mathrm{~mL} / \mathrm{h}$. Airflow was measured with the pneumotachograph of the ventilator, calibrated by an electronic mass flow meter (model 8270, Matheson Gas Products, Montgomeryville, PA, U.S.A.). Tidal volume was calculated by integrating flow. Paw was measured at the connector of the endotracheal tube and Pe through a fluid-filled $5 \mathrm{~F}$ feeding tube with its tip placed into the distal esophagus. All pressure transducers (Sorenson Transpac, Abbott Critical Care Systems, North Chicago, IL, U.S.A.) were calibrated using a water or mercury manometer. Immediately before data acquisition, correct placement of the esophageal tube was checked by performing end-inspiratory airway occlusions and comparing Paw and Pe. A $\Delta \mathrm{Pe} / \Delta \mathrm{Paw}$ ratio of $1.00 \pm 0.05$ was accepted (21). Otherwise, the esophageal catheter was repositioned until correct placement was confirmed. The catheter was continuously flushed with water $(3 \mathrm{~mL} / \mathrm{h})$ to avoid bubble formation. Arterial $\mathrm{Hb}$ oxygen saturation was measured transcutaneously with a Nellcor N 200 pulse oximeter (Nellcor Inc., Hayward, CA, U.S.A.). All signals were digitized at a frequency of $100 \mathrm{~Hz}$ and recorded simultaneously using a data acquisition system (DATAQ Instruments, Inc., Akron, OH, U.S.A.).

Meconium was obtained from healthy human infants less than $24 \mathrm{~h}$ old, pooled, homogenized, and diluted to a 33\% (by weight) solution and stored frozen in $20-\mathrm{mL}$ aliquots until needed.

Protocol. The present study was designed as a randomized controlled trial. After instrumentation, $\mathrm{FiO}_{2}$ was increased to 1.0 and MA was induced by tracheal application of $4 \times 1$ $\mathrm{mL} / \mathrm{kg}$ b.w. meconium solution in three different positions (supine, left lateral, right lateral and supine position). After taking the first set of measurements (time, $30 \mathrm{~min}$ ), animals were switched to the unloading mode of ventilation by using an $\mathrm{FiO}_{2}$ of 1.0 and a PEEP of $0.4 \mathrm{kPa}\left(4 \mathrm{~cm} \mathrm{H}_{2} \mathrm{O}\right)$ for the complete study. Resistive unloading was only applied during inspiration. Resistive unloading was then adjusted to compensate approximately for the expected resistance of the endotracheal tube, which was estimated from peak airflow and previously published data (22). Elastic unloading was adjusted to maintain $\mathrm{PaCO}_{2}$ within the target range. After allowing $30 \mathrm{~min}$ for stabilization, the animals were randomized (time, $0 \mathrm{~min}$ ) by 
using sealed envelopes to receive GV $(n=12)$ or PLV $(n=13)$. In PLV animals, $25 \mathrm{~mL} / \mathrm{kg}$ of prewarmed $\left(38^{\circ} \mathrm{C}\right)$ PFC (Rimar 101, Miteni, Italy) was instilled continuously at a rate of $1 \mathrm{~mL} \cdot \mathrm{kg}^{-1} \cdot \mathrm{min}^{-1}$ into the endotracheal tube without disconnecting the ventilator. The $\mathrm{Kr}$ was increased during the administration of the PFC to compensate for increased airway resistance caused by the presence of liquid in the central airways during filling. The Ke was readjusted if necessary to maintain the $\mathrm{PaCO}_{2}$ within target range. Specifically, the Ke was increased if RR increased by more than $50 \%$ or increased above 120 breaths/min. It could be expected from preliminary experience that this increase is usually associated with $\mathrm{CO}_{2}$ retention. The Ke was decreased if tidal volume was larger than $10 \mathrm{~mL} / \mathrm{kg}$ b.w. because this usually resulted in low RR, a low $\mathrm{PaCO}_{2}$, and eventually apnea. An automatic backup rate with the same ventilator settings as used during induction of MA was applied in case of apnea of more than 5-s duration.

The filling condition was ascertained by disconnecting the animal from the ventilator and performing a slight thoracic compression to observe a liquid meniscus in the endotracheal tube. We aimed for a filling condition with a meniscus close to the anterior chest wall at ambient pressure. Endotracheal-tube suctioning was performed in all animals every $30 \mathrm{~min}$. Evaporative losses of PFC in PLV animals were replaced as follows: If a meniscus was visible at the endotracheal tube after disconnection from the ventilator circuit, only the PFC suctioned was replaced. If there was no meniscus visible but PFC could be suctioned, the PFC suctioned plus $4 \mathrm{~mL} / \mathrm{kg}$ b.w. was replaced. If no PFC was suctioned, $8 \mathrm{~mL} / \mathrm{kg}$ b.w. was replaced. Ten $\mathrm{mL} / \mathrm{kg}$ b.w. of normal saline was infused i.v. in $10 \mathrm{~min}$ for maximal three times whenever the systolic arterial blood pressure dropped below $6.7 \mathrm{kPa}(50 \mathrm{~mm} \mathrm{Hg})$. No inotropic agents and no buffer base correction for acidosis were given throughout the experiment.

Animals were supported until they died spontaneously or were sacrificed after $300 \mathrm{~min}$ with a thiopental overdose (50 $\mathrm{mg}$ ). Immediately after death, the chest was opened, a cannula was placed in the pulmonary artery, the left atrium was opened, and the lung was perfused in situ with Ringer's lactate containing procaine $(250 \mathrm{mg} / \mathrm{L})$, heparin $(20 \mathrm{U} / \mathrm{mL})$, and $\mathrm{CaCl}(2.2$ $\mathrm{mmol} / \mathrm{L})$ for $10 \mathrm{~min}$. Paw was increased to $2 \mathrm{kPa}\left(20 \mathrm{~cm} \mathrm{H}_{2} \mathrm{O}\right)$ for $15 \mathrm{~s}$ and then left at $1 \mathrm{kPa}\left(10 \mathrm{~cm} \mathrm{H}_{2} \mathrm{O}\right)$. Thereafter, the lungs were perfused with a formaldehyde $(4.6 \%)$ /glutaraldehyde $(0.5 \%)$ solution for $10 \mathrm{~min}$. Finally, the lungs were removed and submersed in the same solution, maintaining Paw $=1 \mathrm{kPa}\left(10 \mathrm{~cm} \mathrm{H}_{2} \mathrm{O}\right)$ for at least $8 \mathrm{~h}$.

Data analysis. Data were analyzed during the time 30-300 min after randomization. $\mathrm{PaO}_{2}$ was the primary outcome measure. The following variables were defined as secondary outcome measures: $\mathrm{PaCO}_{2}$ and $\mathrm{pH}$, mortality and incidence of pneumothorax diagnosed at the time of autopsy, inspiratory $\dot{\mathrm{V}}$, tidal volume, RR, MAP, heart rate, cardiac output, and mean arterial blood pressure, mean pulmonary arterial and central venous pressure as average values from the last $10 \mathrm{~min}$ of each 30-min interval. Lung compliance and airway resistance were measured in 10 randomly selected breaths during the same 10-min period and were calculated by a computer program based on the equation of motion (23). WOB on the lung was
Table 1. Baseline data after MA was induced

\begin{tabular}{lcrr}
\hline & GV & PLV & \\
& \multicolumn{1}{c}{$(n=12)$} & \multicolumn{1}{c}{$(n=13)$} & \multicolumn{1}{c}{$p$} \\
\hline Weight $(\mathrm{g})$ & $2893 \pm 168$ & $2941 \pm 181$ & 0.50 \\
Lung compliance $(\mathrm{mL} / \mathrm{kPa} / \mathrm{kg})$ & $4.5 \pm 0.5$ & $4.7 \pm 0.9$ & 0.54 \\
Airway resistance $(\mathrm{kPa} / \mathrm{L} / \mathrm{s})$ & $4.5 \pm 1.1$ & $4.3 \pm 1.4$ & 0.77 \\
$\mathrm{PaO}_{2}(\mathrm{kPa})$ & $21.7 \pm 9.2$ & $19.7 \pm 6.1$ & 0.51 \\
$\mathrm{Mean}_{\text {arterial blood } \mathrm{pressure}(\mathrm{kPa})}$ & $6.8 \pm 1.5$ & $6.8 \pm 0.8$ & 0.99 \\
Cardiac output $(\mathrm{mL} / \mathrm{kg} / \mathrm{min})$ & $179 \pm 34$ & $150 \pm 24$ & 0.046 \\
\hline
\end{tabular}

Values are mean $\pm \mathrm{SD}$.

calculated as the area given by the integral of inspiratory transpulmonary pressure over volume using the same breaths. Transpulmonary pressure was defined as the difference between Paw and Pe. The power of breathing was calculated as WOB over time. MAP was calculated as the integral of Paw divided by the recording time. Arterial blood gases were measured every 30 min using an automated blood gas analyzer (AVL, Compact 2, Bad Homburg, Germany). $\mathrm{SaO}_{2}$ was calculated taking into account $\mathrm{PO}_{2}, \mathrm{PCO}_{2}$, and $\mathrm{pH}$, assuming a $\mathrm{P}_{50}$ of $26.7 \mathrm{~mm} \mathrm{Hg}$. The shunt fraction was calculated as $\{[1.34 \cdot \mathrm{Hb}+$ $0.003 \cdot\left(717-52-\mathrm{PaCO}_{2}[\mathrm{~mm} \mathrm{Hg}]-\mathrm{PFC}\right.$-vapor pressure $\left.)\right]-$ $\left.\left(1.34 \cdot \mathrm{Hb} \cdot \mathrm{SaO}_{2}[\%] / 100+0.003 \cdot \mathrm{PaO}_{2}[\mathrm{~mm} \mathrm{Hg}]\right)\right\} /\{[1.34 \cdot \mathrm{Hb}$ $+0.003 \cdot\left(717-52-\mathrm{PacO}_{2}[\mathrm{~mm} \mathrm{Hg}]-\mathrm{PFC}\right.$-vapor pressure $\left.)\right]$ $\left.-\left(1.34 \cdot \mathrm{Hb} \cdot \mathrm{Svo}_{2}[\%] / 100+0.003 \cdot \mathrm{Pvo}_{2}[\mathrm{~mm} \mathrm{Hg}]\right)\right\}$, where the mean atmospheric pressure is $717 \mathrm{~mm} \mathrm{Hg}$, water and PFC-vapor pressures at $39^{\circ} \mathrm{C}$ are 52 and $64 \mathrm{~mm} \mathrm{Hg}$, respectively, and $\mathrm{SvO}_{2}$ is mixed venous saturation. Cardiac output was measured every $30 \mathrm{~min}$ by i.v. injection of ice-cold normal saline three times and averaged at each time point.

Lung histology. Slides were obtained from dependent and nondependent parts of both lungs in each animal, stained with hematoxylin/eosin, and evaluated by a pathologist (R.S.) blinded to the animal's group assignment by using a modified previously described score (24). Variables scored were atelectasis, alveolar and interstitial inflammation, alveolar and interstitial hemorrhage, alveolar and interstitial edema, necrosis, and overdistension. Each variable was scored by using a $0-4$-point scale with no injury $=0$, injury in $25,50,75 \%$ and throughout the field scoring 1, 2, 3, and 4 .

Statistics. Differences of categorical variables between groups were analyzed by using Fisher's exact test and of continuous variables by 2-tailed $t$ tests, Mann-Whitney U tests, and ANOVA for repeated measurements where appropriate. Missing data points secondary to early deaths were handled using a general linear model (Sigmastat V. 2.03, SPSS Inc., Chicago, IL, U.S.A.). Values of $p<0.05$ were considered significant. Values are presented as mean \pm SD or median

Table 2. Ketamine dose and ventilator settings during the study period

\begin{tabular}{lcc}
\hline & $\begin{array}{c}\text { GV } \\
(n=12)\end{array}$ & $\begin{array}{c}\text { PLV } \\
(n=13)\end{array}$ \\
\hline Ketamine dose $(\mathrm{mg} / \mathrm{kg} / \mathrm{h})$ & $75 \pm 12$ & $65 \pm 20$ \\
Elastic unloading $(\mathrm{Pa} / \mathrm{mL})$ & $62 \pm 5$ & $48 \pm 12 \dagger$ \\
Resistive unloading $(\mathrm{kPa} / \mathrm{L} / \mathrm{s})$ & $5.4 \pm 1.4$ & $5.5 \pm 1.0$ \\
\hline
\end{tabular}

Values are mean $\pm \mathrm{SD} ; \dagger=p<0.01$. Values given were obtained by first calculating individual mean values for each animal across the experimental period, followed by calculating mean \pm SD across each group. 
(A)

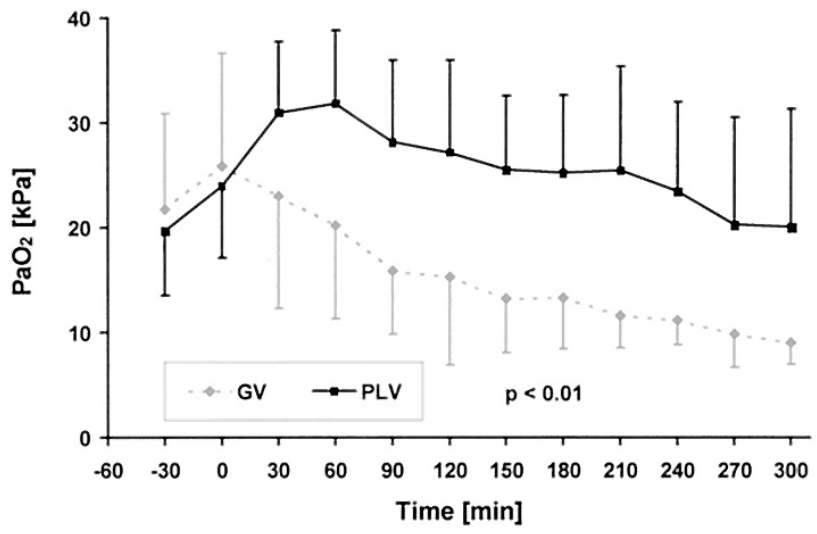

(C)

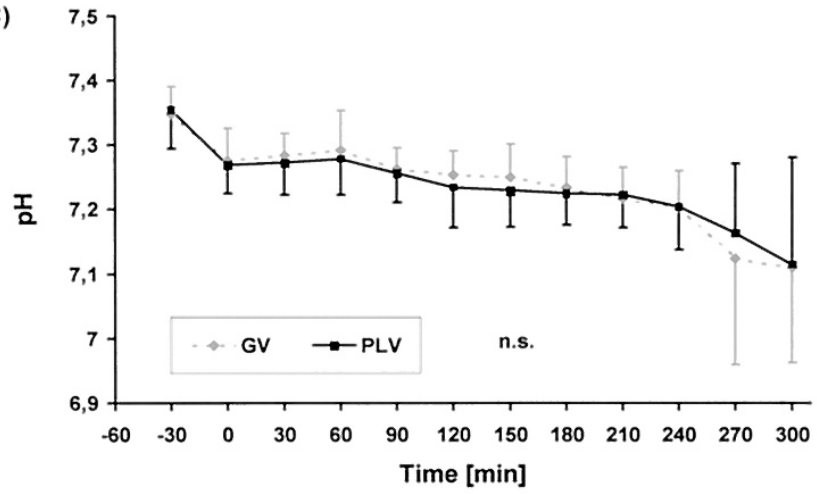

(B)

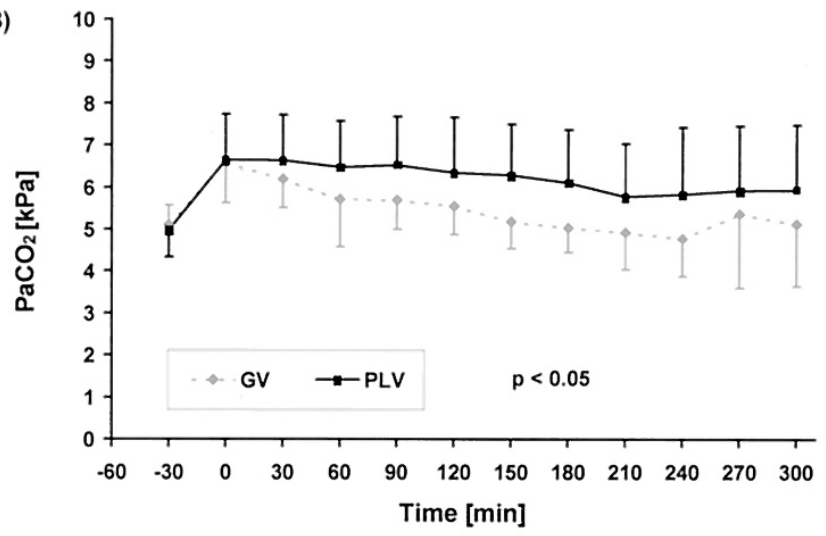

(D)

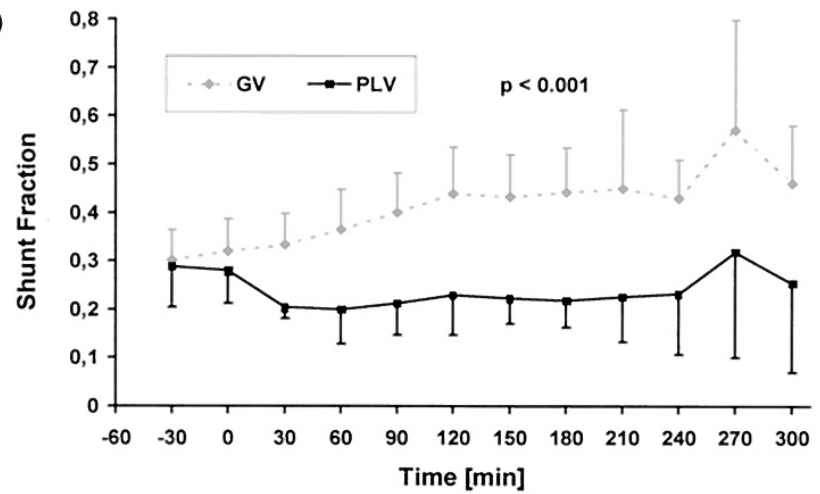

Figure 1. Arterial blood gas measurements: $\mathrm{PaO}_{2}(A), \mathrm{PaCO}_{2}(B), \mathrm{pH}(C)$, and shunt fraction $(D) \dagger$ during GV and PLV. Values are mean $\pm \mathrm{SD} ; \dagger n=9$ in each group.

(minimum-maximum). A sample-size calculation revealed that 21 animals would be necessary to detect a $6.7-\mathrm{kPa}(50 \mathrm{~mm} \mathrm{Hg})$ difference in $\mathrm{PaO}_{2}$ between groups $[\alpha=0.05, \beta=0.2$, expected SD for $\mathrm{PaO}_{2}$ measurements: $\left.5.3 \mathrm{kPa}(40 \mathrm{~mm} \mathrm{Hg})\right]$. To compensate for potential losses before randomization, sample size was increased by $20 \%$ to 25 animals.

\section{RESULTS}

All 25 animals survived MA induction and were randomized. The two groups were similar regarding their baseline characteristics except for cardiac output, which was higher in the GV group before randomization (Table 1). Instillation of PFC was well tolerated in all animals except for brief apnea in nine of the 13 PLV animals, occurring mainly during the beginning of instillation and sometimes requiring brief episodes of backup rate ventilation. Otherwise, this backup rate was not switched on except during final apnea in animals dying before the end of the study period. The filling condition was assessed by regular suctioning procedures, and PFC was suctioned from the main airways in $72 \%$ of all procedures performed. In $28 \%$ of all procedures performed, no PFC could be suctioned, suggesting a somewhat lower filling condition. Coughing was induced in one PLV animal during filling but disappeared immediately after an additional i.v. injection of 10 mg ketamine. Thereafter, we did not observe spontaneous coughing other than brief and self-limited episodes of coughing during the suctioning procedures. The ketamine dose and the
Kr were similar during both ventilatory conditions, whereas the Ke was higher during GV versus PLV (Table 2).

Gas exchange and ventilation. Whereas $\mathrm{PaO}_{2}$ increased during PFC instillation, it declined continuously in the GV group and was significantly lower during GV compared with PLV (Fig. 1A). The shunt fraction was lower during PLV (Fig. $1 D$ ). $\mathrm{PaCO}_{2}$ was lower during GV (Fig. $1 B$ ), whereas there was no significant difference in $\mathrm{pH}$ (Fig. $1 C$ ). The lower $\mathrm{PaCO}_{2}$ during GV was caused by a larger $\dot{V}$ (Fig. 2A). This was secondary to a higher RR (Fig. $2 B$ ), with no significant difference in tidal volumes between groups (Fig. $2 C$ ). There was a trend toward a higher MAP in GV animals toward the end of the study period (Fig. 2D).

Respiratory mechanics. Whereas there was no change in compliance over time in GV animals, compliance improved during liquid instillation and remained significantly higher (Fig. 3A). Resistance was higher at time $30 \mathrm{~min}$ in PLV animals, as this time point just included the final phase of PFC instillation. Subsequently there was no large difference between groups, but if analyzed over time, resistance was significantly lower in GV animals (Fig. 3B). WOB (Fig. 3C) and power of breathing (Fig. $3 D$ ) were significantly higher in $\mathrm{GV}$ versus PLV animals.

Hemodynamics. The pulmonary arterial catheter could be placed successfully in nine animals in each group. There was no difference in mean arterial blood pressure, central venous and mean pulmonary arterial pressure, and heart rate (Fig. 4). 
(A)

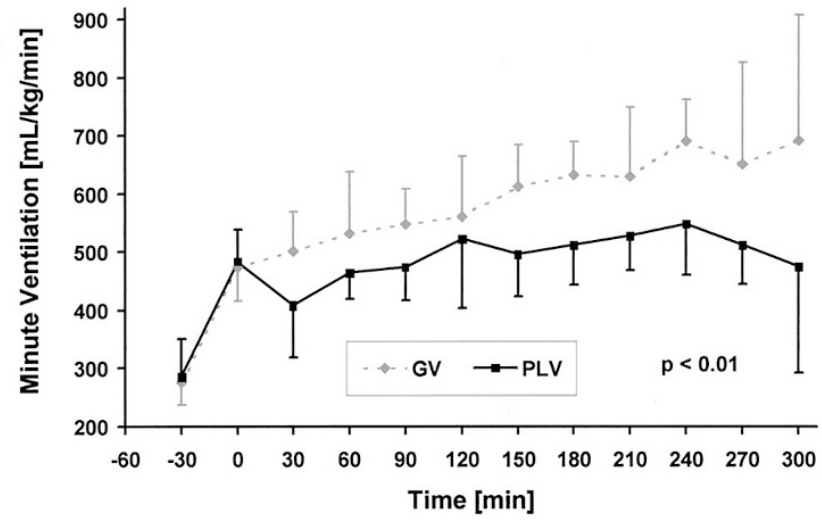

(C)

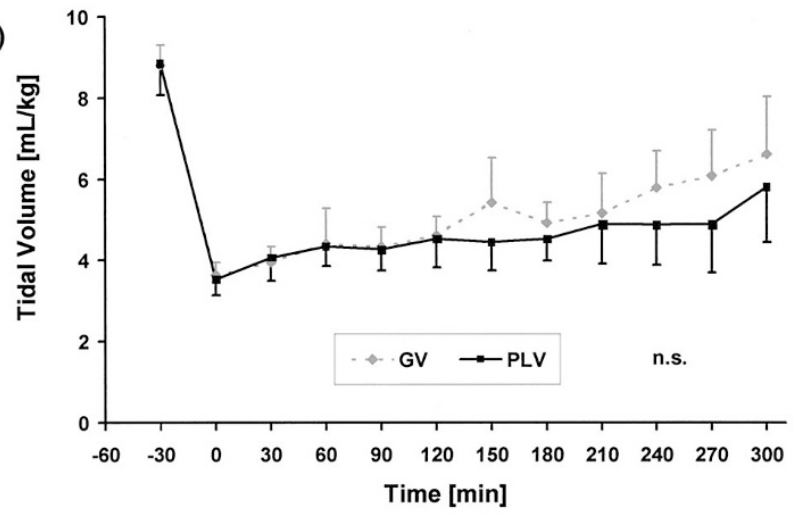

(B)

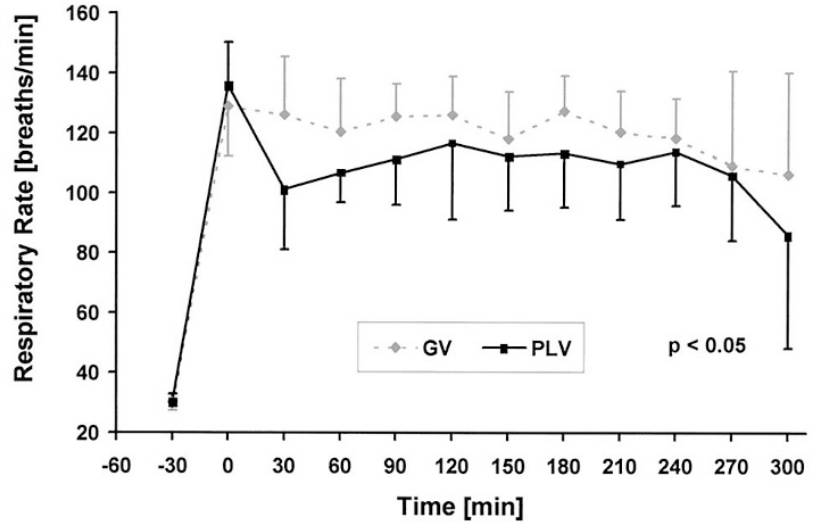

(D)

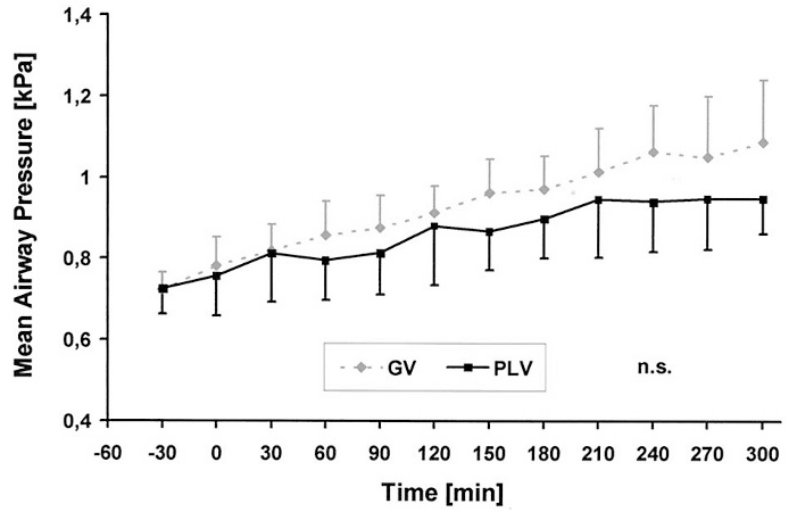

Figure 2. Minute ventilation $(A), \operatorname{RR}(B)$, tidal volume $(C)$, and MAP $(D)$ during GV and PLV. Values are mean \pm SD.

Cardiac output was higher during GV (Fig. 4E), but this difference was present before randomization, and, when data were analyzed as a percentage of baseline measurements (time 0 ), there was no difference between groups (values available on request).

Mortality, incidence of pneumothorax, and lung histology. Five of the $12 \mathrm{GV}$ compared with one of the 13 PLV animals died before the end of the study period $(p<0.05)$. There was no difference in the incidence of pneumothorax [7/12 (58\%) versus 6/13 (46\%); GV versus PLV)]. The results of the histologic evaluation are shown in Table 3. Scores were obtained from four sites in each animal's lungs. Because there were no differences between left versus right lung and dependent versus nondependent areas, an average score was calculated for each variable in each animal as well as a sum score in each animal. Scores for alveolar and interstitial inflammation, alveolar and interstitial hemorrhage, and atelectasis as well as the sum score were higher in GV compared with PLV animals, indicating more lung injury in GV animals. No differences in histologic scores were found in GV animals that died before the end of the experimental period versus those that survived (data not presented). Figure 5, $A$ and $B$, shows typical examples of lung histology of one animal of each group.

\section{DISCUSSION}

It has been shown previously that meconium interferes with surfactant function $(25,26)$. Whereas some experimental studies $(27,28)$ and uncontrolled case series in human infants $(29)$ suggest a beneficial effect of exogenous surfactant treatment, others do not (30). Because PLV may reduce interfacial surface tension in the alveoli, it may help to recruit atelectasis and improve ventilation-perfusion mismatch. Furthermore, PFC within the airways may help to mobilize meconium to be removed by suction procedures.

Shaffer et al. (31) have shown in a cohort study that lambs with MA may be supported effectively using total liquid ventilation for $90 \mathrm{~min}$ and that oxygenation and pulmonary mechanics remain significantly improved after returning animals to GV. Foust et al. (3) showed in a randomized controlled experimental study in rabbits that PLV as well as total liquid ventilation resulted in improved oxygenation. Other authors reported similar findings in a piglet model of MA using PLV (32-34). Whereas all of these studies have been done using controlled ventilation in paralyzed animals, our ventilatory approach aims at maintaining spontaneous breathing to avoid possible side effects of muscle paralysis. The principal finding of our study is that $\mathrm{PaO}_{2}$ is improved during PLV in spontaneously breathing rabbits with severe MA when supported by respiratory mechanical unloading. We have found previously that the same ventilatory approach results in impaired oxygenation in animals without lung disease (13), and this former finding may be explained by impaired diffusion, ventilationperfusion heterogeneity, and shunt secondary to the PFC being present inside the alveoli (35). Therefore, the improved $\mathrm{PaO}_{2}$ in our animals with MA can only be explained by significant recruitment of collapsed alveoli resulting in improved ventila- 
(A)

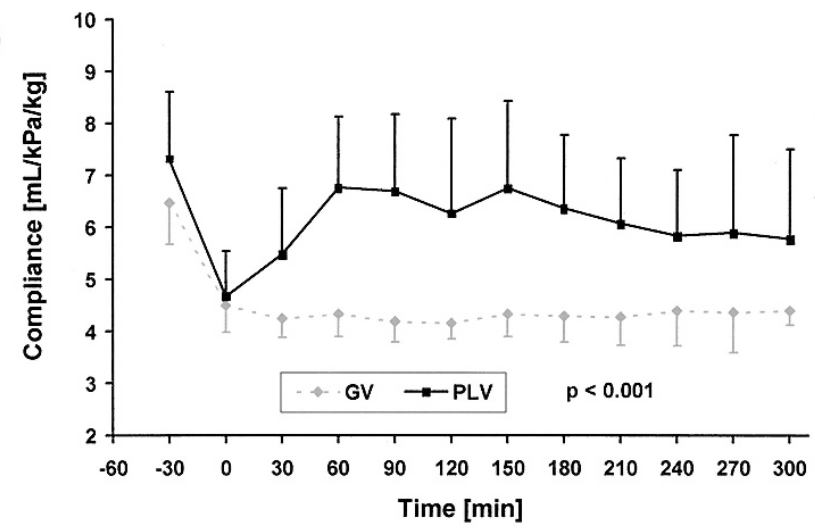

(C)

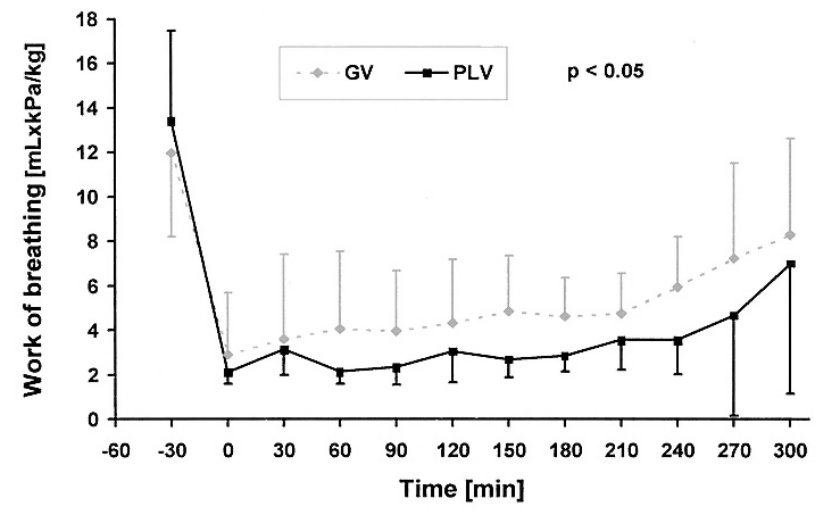

(B)

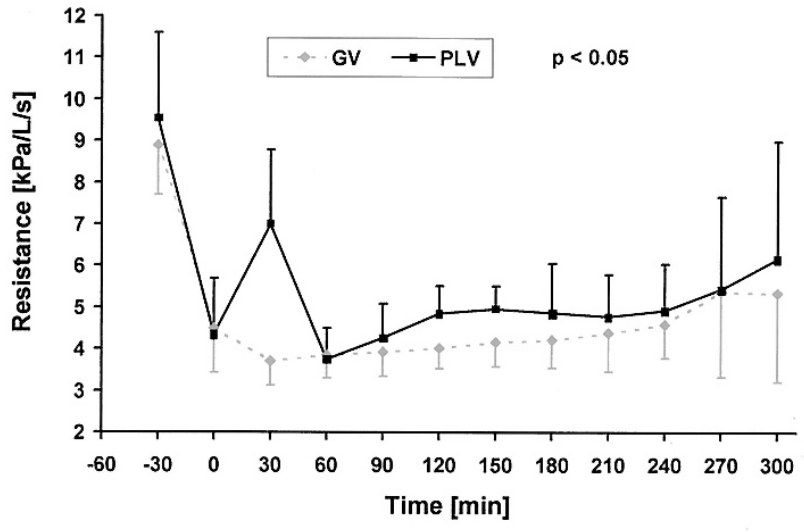

(D)

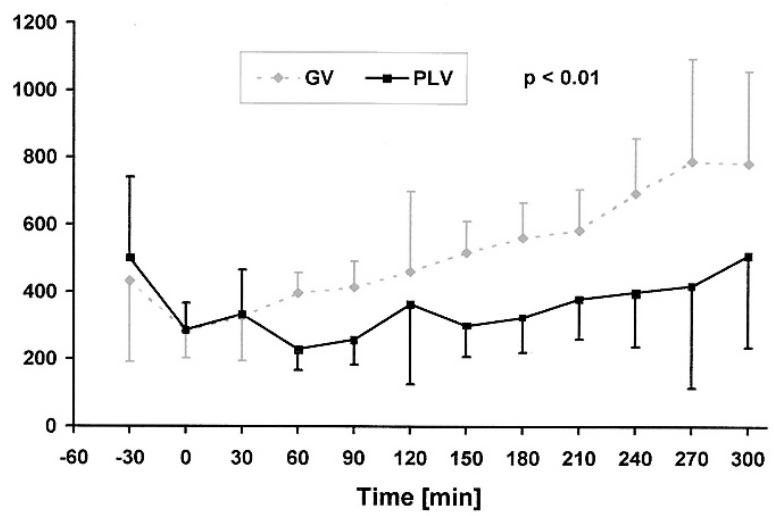

Figure 3. Compliance $(A)$, resistance $(B)$, WOB $(C)$, and power of breathing $(D)$ during GV and PLV. Values are mean $\pm \mathrm{SD}$.

tion/perfusion match and decreased shunt. $\mathrm{PaCO}_{2}$ was lower by approximately $6 \mathrm{~mm} \mathrm{Hg}$ in the GV group secondary to the larger $\dot{V}$, which was mainly due to the higher $R R$ in these animals. This difference cannot be explained by differences in anesthesia levels because there was no difference in ketamine dosage. The higher respiratory drive in GV animals may also explain the trend toward a higher MAP toward the end of the experimental period because ventilatory support and patient effort are coupled in our ventilator system. The higher $\mathrm{PaCO}_{2}$ values in the PLV group may also be caused at least in part by impaired diffusion in the presence of PVC within the alveoli (35). Lung compliance improved during liquid instillation, resulting in larger tidal volumes and lower RR sometimes within a few minutes after beginning the instillation of PFC. This effect allowed the reduction of ventilatory support, which is reflected by the decreased $\mathrm{Ke}$ and a trend toward a lower MAP in PLV animals. This is in contrast to Foust et al. (3) who found an improvement in compliance only in the tidal liquid ventilation group but not in the PLV group. The higher RR in our animals may account at least in part for the different findings. Measurement of lung mechanics usually does not account for inertial forces because they are considered negligible. However, this may result in systematic errors in measurements of dynamic lung compliance, especially at high RR (36), and these errors may be even more pronounced during liquid ventilation because of the high specific gravity of this respiratory medium causing higher inertial forces than during GV. Resistance was slightly but significantly higher in the PLV compared with the GV group in our study, which may be explained by some liquid flushing back to the airways during expiration, the resistive load of the liquid itself, and/or by higher inertial forces.

Because PFC have a higher specific gravity and a much higher viscosity compared with gas, they result in increased workload in subjects without lung disease (13). In PLV animals, the power of breathing was reduced, which is in part secondary to the lower $\dot{V}$. However, WOB, which reflects the workload on the lung of every single breath, was also reduced, although there was no difference in tidal volume between groups. This suggests that there was a true reduction of the workload on the lung in PLV animals, which was caused by the improved lung compliance.

We did not find differences in heart rate or in systemic and pulmonary arterial or central venous pressure between groups. However, there was a trend toward a lower systemic arterial pressure in the GV group. Furthermore, we observed a trend toward an increasing pulmonary arterial pressure in GV animals that is consistent with observations from Foust et al. (3) and suggests increased pulmonary vascular resistance. Cardiac output was significantly lower in the PLV group. However, this difference was observed during the entire experimental period and was present already at the time before MA was induced and at the time of randomization. If cardiac output was expressed as a percentage of baseline measurements, there was no difference between groups. Therefore, we believe that PLV did not impair cardiac output. Other investigators did not find any difference comparing hemodynamics in an MA piglet model between liquid and GV groups $(32,33)$. 
(A)

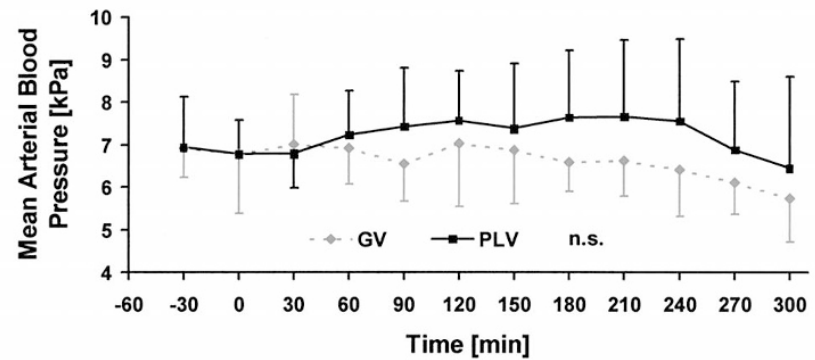

(B)

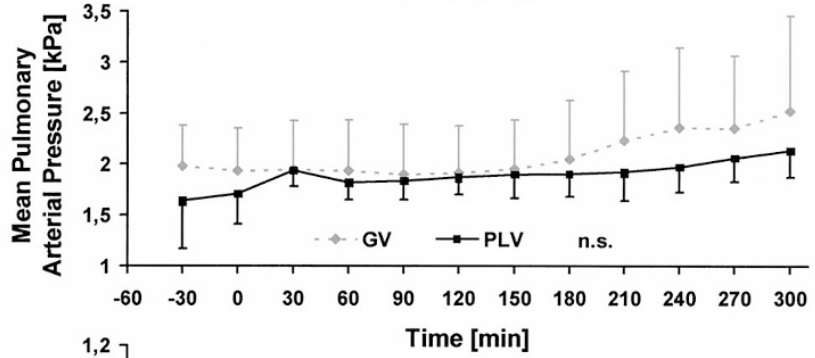

(C)

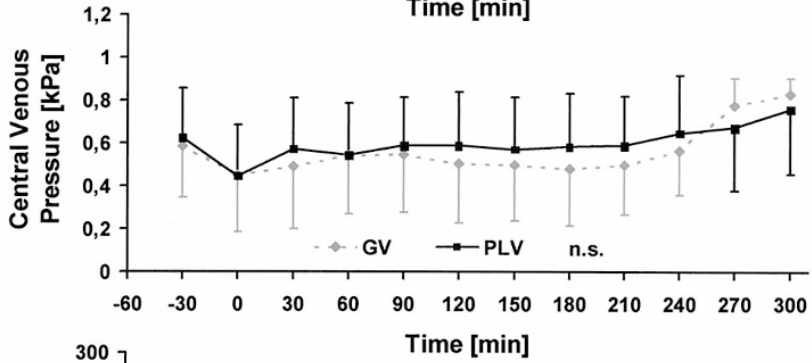

(D)

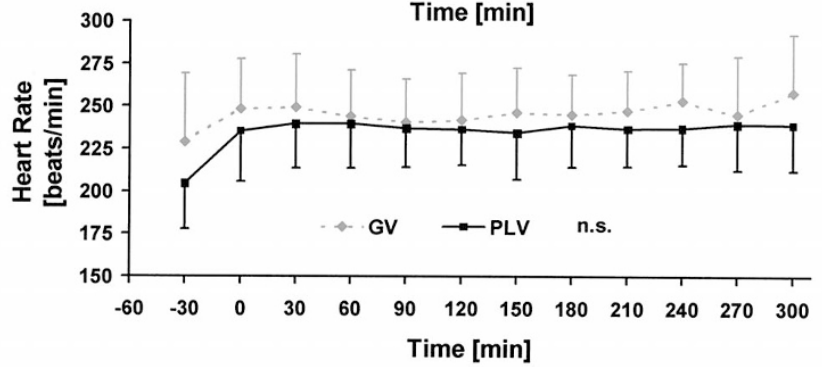

(E)

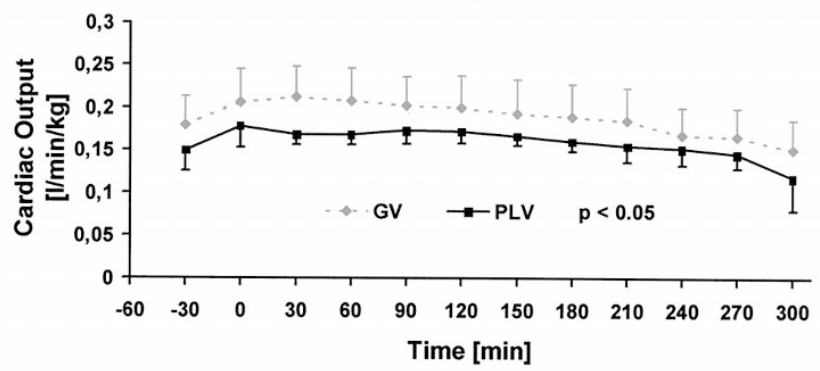

Figure 4. Hemodynamic variables: mean arterial blood pressure $(A)$, mean pulmonary arterial pressure $(B) \dagger$, central venous pressure $(C)$, heart rate $(D)$, and cardiac output $(E) \dagger$. Values are mean $\pm \mathrm{SD} ; \dagger n=9$ in each group.

Mortality was significantly reduced in the PLV group. The only observed cause of death before the end of the experimental period was pneumothorax. Pneumothorax was not treated in both groups and was diagnosed at autopsy. However, only one animal of the GV group with a pneumothorax survived until the end of the study period, whereas four of the PLV group did. Because we did not quantitate the amount of air or PFC within the pleural space at autopsy, we can only speculate that the degree of lung damage and leakage of air or PFC may have been less in PLV animals compared with GV animals. The observed incidence of pneumothorax is higher than reported
Table 3. Lung Histology

\begin{tabular}{lll}
\hline & \multicolumn{1}{c}{ GV $(n=12)$} & PLV $(n=13)$ \\
\hline Atelectasis & $1.38(0.5-2.0)$ & $1.0(0.25-1.25)^{*}$ \\
Alveolar inflammation & $2.0(1.25-3.0)$ & $1.25(1.0-2.0) \dagger$ \\
Interstitial inflammation & $2.0(1.0-2.5)$ & $1.25(1.0-1.75) \dagger$ \\
Alveolar hemorrhage & $1.13(0.5-2.0)$ & $0.25(0-1.0) \dagger$ \\
Interstitial hemorrhage & $1.0(0-1.5)$ & $0.75(0-1.0)^{*}$ \\
Alveolar edema & $0.5(0-2.25)$ & $0(0-1.75)$ \\
Interstitial edema & $1.0(1.0-1.25)$ & $1.0(1.0-1.0)$ \\
Necrosis & 0 & 0 \\
Overdistension & $1.5(0.75-2.0)$ & $1.5(0.75-2.25)$ \\
\hline Sum & $11.13(7.25-13.75)$ & $7.5(5.5-10.0) \dagger$ \\
\hline
\end{tabular}

Values are median (minimum-maximum); ${ }^{*} p<0.05 ; \dagger p<0.01$. Scores were obtained from four sites in each animal's lungs. Because there were no differences between right vs left lung and dependent vs nondependent areas, an average score was calculated for each variable in each animal as well as a sum score in each animal.
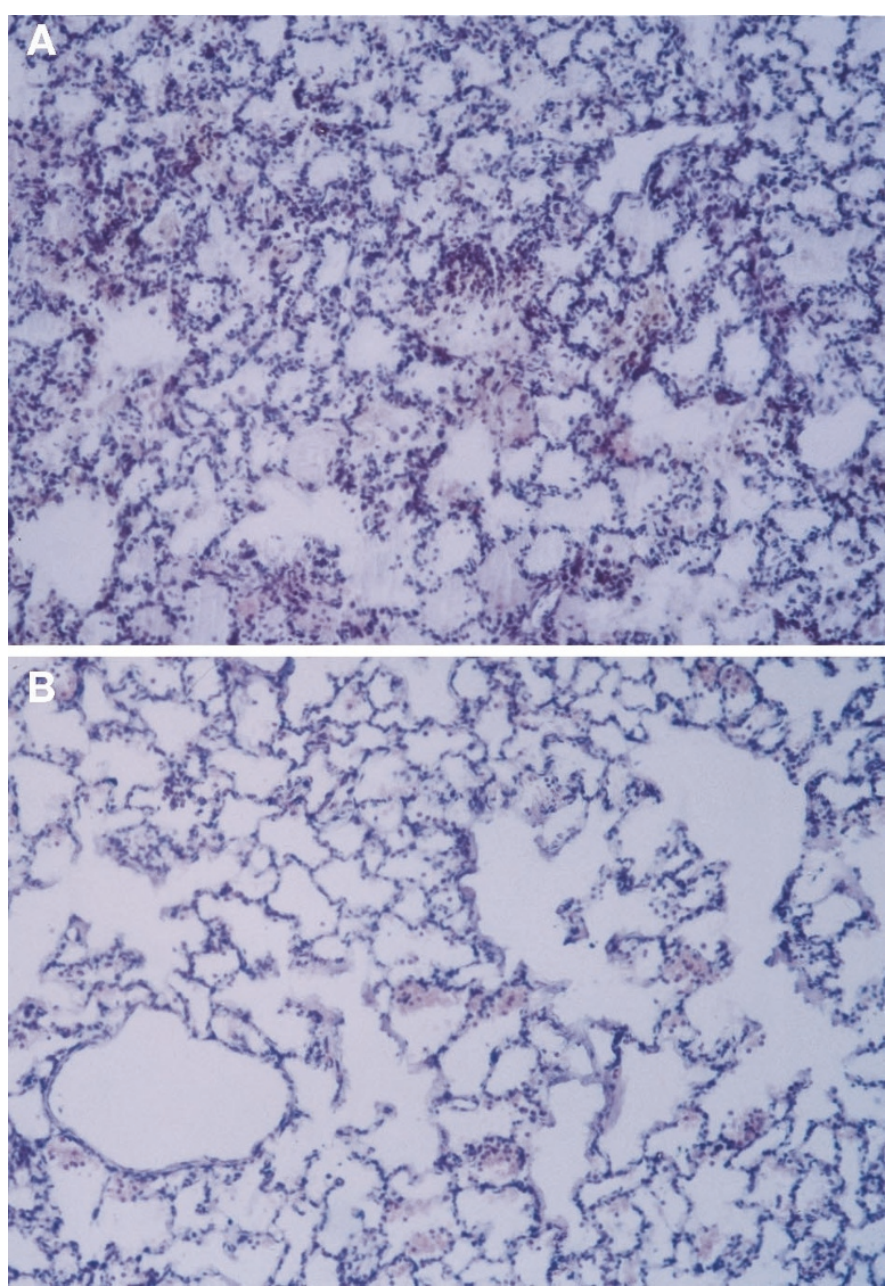

Figure 5. Histology $(\times 40$ original size $): G V(A)$ vs PLV $(B)$. Typical examples: in GV animals, more atelectasis with signs of inflammation (granulocytes) and hemorrhage were found; in PLV animals, a better and more even aeration and fewer signs of inflammation and hemorrhage were found.

from other studies $(3,27)$. It may be speculated that the high RR observed in our animals may not have allowed for an expiratory time long enough for complete exhalation, resulting in air trapping, which would be a significant disadvantage of the type of ventilatory support used in this study. A comparison 
between conventional mechanical ventilation and paralysis and our ventilatory approach during PLV is necessary to clarify this issue.

Histologic evaluation revealed higher scores for alveolar and interstitial inflammation, hemorrhage, and atelectasis and explains the lower compliance found in GV animals. Other investigators (3) have found a more even aeration in PLVtreated animals with MA compared with GV. This is consistent with our finding of less atelectasis in PLV animals. A more even aeration in PLV animals may result in less overdistension of open alveoli and, therefore, less volutrauma and lung injury. This is further supported by the lower degree of inflammation and hemorrhage in PLV animals, suggesting less lung injury. Another possible explanation of these findings could be a more effective removal of meconium from the airways during the regular suction procedure in PLV animals (32). However, it was our impression that little meconium was recovered during the suctioning procedures in both groups. Because we did not measure meconium recovery, we can only speculate that this factor may not have played an important role in the prevention of lung injury in PLV animals. Other studies found evidence that PFC may protect the lung from oxidative damage (37), reduce neutrophil accumulation (38), and protect lung epithelial cells from neutrophil-mediated injury (39). This protective effect may have contributed to reduce the degree of lung injury in our PLV group.

Our results add to the growing body of evidence that PLV may be successfully used in spontaneously breathing subjects with severe lung disease, allowing usage of spontaneous respiratory effort and avoiding muscle paralysis with its side effects. The ventilatory support used in our study may be perfectly suited to support spontaneously breathing subjects undergoing PLV, because it allows one to selectively compensate for decreased compliance and increased resistance. However, the approach to adjust the $\mathrm{Kr}$ mainly to a level compensating for the imposed resistance of the endotracheal tube may have been suboptimal for the PLV group and may be responsible for the observed difference in $\mathrm{PaCO}_{2}$ between groups.

The study protocol for the PLV group aimed for the large airways being filled with gas when exposed to PEEP and with PFC when exposed to ambient pressure. This filling condition was assessed by regular suctioning procedures and was confirmed in $72 \%$ of all procedures performed. However, resistance was slightly elevated in the PLV group, which may be related to the presence of some PFC within the airways when exposed to PEEP. We speculate that frequent measurements of pulmonary mechanics and adjustments of the degree of both types of unloading may have resulted in higher $\mathrm{Kr}$ in the PLV group and a better match in $\mathrm{PaCO}_{2}$. The higher $\mathrm{PaCO}_{2}$ in the PLV group may have limited the observed difference in $\mathrm{PaO}_{2}$ between groups or affected hemodynamics and, therefore, may have influenced some of the study results. However, given the magnitude of the observed average difference in $\mathrm{PaCO}_{2}(0.8 \mathrm{kPa}$ $=6 \mathrm{~mm} \mathrm{Hg}$ ), we speculate that this difference would not affect outcome measurements to a significant degree.

The animal model used is not a neonatal model, and some of the study findings may be different in the developing lung if a neonatal model such as the newborn piglet is studied. For example, the effects of MA on the pulmonary vasculature may be more pronounced in the adapting neonatal lung. On the other hand, the neonatal heart may tolerate a sudden increase in right ventricular afterload better than the heart of an adult subject.

In conclusion, we have shown that PLV improves oxygenation and compliance and may improve survival in spontaneously breathing rabbits with severe MA supported by PAV. It may result in less lung injury compared with GV without significant negative effects on hemodynamics. Further prospective studies are needed to confirm the impact of this mode of ventilation on survival and lung injury and to clarify whether or not spontaneous breathing has any significant physiologic or long-term clinical advantages compared with controlled mechanical ventilation in paralyzed subjects with a partially liquid-filled lung.

Acknowledgments. The authors thank Prof. B. Jilge for generously supporting this study, Dr. B. Kuhnt for her expert technical assistance and advice, and Nelson Claure (University of Miami) for his expert technical support.

\section{REFERENCES}

1. Leach CL, Holm B, Morin III FC, Fuhrman BP, Papo MC, Steinhorn D, Hernan LJ 1995 Partial liquid ventilation in premature lambs with respiratory distress syndrome: efficacy and compatibility with exogenous surfactant. J Pediatr 126:412-420

2. Leach CL, Fuhrman BP, Morin III FC, Rath MG 1993 Perfluorocarbon-associated gas exchange (partial liquid ventilation) in respiratory distress syndrome: a prospective, randomized, controlled study. Crit Care Med 21:1270-1278

3. Foust III R, Tran NN, Cox C, Miller TF, Greenspan JS, Wolfson MR, Shaffer TH 1996 Liquid-assisted ventilation: an alternative ventilatory strategy for acute meconium aspiration injury. Pediatr Pulmonol 21:316-322

4. Leach CL, Greenspan JS, Rubenstein SD, Shaffer TH, Wolfson MR, Jackson JC, DeLemos R, Fuhrman BP 1996 Partial liquid ventilation with perflubron in premature infants with severe respiratory distress syndrome. N Engl J Med 335:761-767

5. Buck ML, Reed MD 1991 Use of nondepolarizing neuromuscular blocking agents in mechanically ventilated patients. Clin Pharm 10:32-48

6. Bhutani VK, Abbasi S, Sivieri EM 1988 Continuous skeletal muscle paralysis: effect on neonatal pulmonary mechanics. Pediatrics 81:419-422

7. Miller J, Law AB, Parker RA, Sundell H, Silberberg AR, Cotton RB 1994 Effects of morphine and pancuronium on lung volume and oxygenation in premature infants with hyaline membrane disease. J Pediatr 125:97-103

8. Putensen C, Mutz NJ, Putensen-Himmer G, Zinserling J 1999 Spontaneous breathing during ventilatory support improves ventilation-perfusion distributions in patients with acute respiratory distress syndrome. Am J Respir Crit Care Med 159:1241-1248

9. Hummler H, Gerhardt T, Gonzalez A, Claure N, Everett R, Bancalari E 1996 Influence of different methods of synchronized mechanical ventilation on ventilation, gas exchange, patient effort, and blood pressure fluctuations in premature infants. Pediatr Pulmonol 22:305-313

10. Cleary JP, Bernstein G, Mannino FL, Heldt GP 1995 Improved oxygenation during synchronized intermittent mandatory ventilation in neonates with respiratory distress syndrome: a randomized, crossover study. J Pediatr 126:407-411

11. Bernstein G, Mannino FL, Heldt GP, Callahan JD, Bull DH, Sola A, Ariagno RL, Hoffman GL, Frantz III ID, Troche BI, Roberts JL, Dela Cruz TV, Costa E 1996 Randomized multicenter trial comparing synchronized and conventional intermittent mandatory ventilation in neonates. J Pediatr 128:453-463

12. Bendel-Stenzel EM, Mrozek JD, Bing D, Meyers PA, Connett JE, Mammel MC 1998 Dynamics of spontaneous breathing during patient-triggered partial liquid ventilation. Pediatr Pulmonol 26:319-325

13. Hummler H, Schulze A, Pohlandt F, Thome U 2000 Dynamics of breathing during partial liquid ventilation in spontaneously breathing rabbits supported by elastic and resistive unloading. Pediatr Res 47:392-397

14. Bendel-Stenzel EM, Bing DR, Meyers PA, Connett JE, Mammel MC 1999 Synchronized gas and partial liquid ventilation in lung-injured animals: improved gas exchange with decreased effort. Pediatr Pulmonol 27:242-250

15. Schaller P, Schulze A 1991 A ventilator generating a positive or negative internal compliance. Upsala J Med Sci 96:219-234

16. Younes M 1992 Proportional assist ventilation, a new approach to ventilatory support Theory. Am Rev Respir Dis 145:114-120

17. Schulze A, Gerhardt T, Musante G, Schaller P, Claure N, Everett R, Gomez-Marin O, Bancalari E 1999 Proportional assist ventilation in low birth weight infants with acute respiratory disease: a comparison to assist/control and conventional mechanical ventilation. J Pediatr 135:339-344 
18. Fuhrman BP, Paczan PR, De Francisis M 1991 Perfluorocarbon-associated gas exchange. Crit Care Med 19:712-722

19. Tütüncü AS, Houmes RJ, Bos JA, Wollmer P, Lachmann B 1996 Evaluation of lung function after intratracheal perfluorocarbon administration in healthy animals. Crit Care Med 24:274-279

20. Schulze A, Rich W, Schellenberg L, Schaller P, Heldt GP 1998 Effects of different gain settings during assisted mechanical ventilation using respiratory unloading in rabbits. Pediatr Res 44:132-138

21. Coates A, Stocks J, Gerhardt T 1996 Esophageal manometry. In: Stocks J, Sly PD, Tepper RS, Morgan WJ (eds) Infant Respiratory Function Testing. Wiley-Liss, New York, pp 241-258

22. Wall MA 1980 Infant endotracheal tube resistance: effects of changing length, diameter, and gas density. Crit Care Med 8:38-40

23. Silva Neto G, Gerhardt T, Silberberg A, Claure N, Duara S, Bancalari E 1992 Nonlinear pressure/volume relationship and measurements of lung mechanics in infants. Pediatr Pulmonol 12:146-152

24. Mrozek JD, Smith KM, Bing DR, Meyers PA, Simonton SC, Connett JE, Mamme MC 1997 Exogenous surfactant and partial liquid ventilation. Physiologic and pathologic effects. Am J Respir Crit Care Med 156:1058-1065

25. Clark DA, Nieman GF, Thompson JE, Paskanik AM, Rokhar JE, Bredenberg CE 1987 Surfactant displacement by meconium free fatty acids: an alternative explanation for atelectasis in meconium aspiration syndrome. J Pediatr 110:765-770

26. Bae CW, Takahashi A, Chida S, Sasaki M 1998 Morphology and function of pulmonary surfactant inhibited by meconium. Pediatr Res 44:187-191

27. Paranka MS, Walsh WF, Stancombe BB 1992 Surfactant lavage in a piglet model of meconium aspiration syndrome. Pediatr Res 31:625-628

28. Cochrane CG, Revak SD, Merritt TA, Schraufstätter IU, Hoch RC, Henderson C Andersson S, Takamori H, Oades Z 1998 Bronchoalveolar lavage with KL4 surfactant in models of meconium aspiration syndrome. Pediatr Res 44:705-715
29. Lam BCC, Yeung CY 1999 Surfactant for meconium aspiration syndrome: a pilot study. Pediatrics 103:1014-1018

30. Wiswell TE, Peabody SS, Davis JM, Slayter MV, Bent RC, Merritt TA 1994 Surfactant therapy and high-frequency jet ventilation in the management of a piglet model of the meconium aspiration syndrome. Pediatr Res 36:494-500

31. Shaffer TH, Lowe CA, Bhutani VK, Douglas PR 1984 Liquid ventilation: effects on pulmonary function in distressed meconium-stained lambs. Pediatr Res 18:47-52

32. Kuo CY, Hsueh C, Wang CR 1998 Liquid ventilation for treatment of meconium aspiration syndrome in a piglet model. J Formos Med Assoc 97:392-399

33. Barrington KJ, Singh AJ, Etches PC, Finer NN 1999 Partial liquid ventilation with and without inhaled nitric oxide in a newborn piglet model of meconium aspiration. Am J Respir Crit Care Med 160:1922-1927

34. Nakamura T, Matsuzawa S, Sugiura M, Tamura M 2000 A randomised control study of partial liquid ventilation after airway lavage with exogenous surfactant in a meconium aspiration syndrome animal model. Arch Dis Child 82:F160-F162

35. Mates EA, Hildebrandt J, Jackson JC, Tarczy-Hornoch P, Hlastala MP 1997 Shunt and ventilation-perfusion distribution during partial liquid ventilation in healthy piglets. J Appl Physiol 82:933-942

36. Lanteri CJ, Petak F, Gurrin L, Sly PD 1999 Influence of inertance on respiratory mechanics measurements in mechanically ventilated puppies. Pediatr Pulmonol 28: $130-138$

37. Steinhorn DM, Papo MC, Rotta AT, Aljada A, Fuhrman BP, Dandona P 1999 Liquid ventilation attenuates pulmonary oxidative damage. J Crit Care 14:20-28

38. Colton DM, Till GO, Johnson KJ, Dean SB, Bartlett RH, Hirschl RB 1998 Neutrophil accumulation is reduced during partial liquid ventilation. Crit Care Med 26:17161724

39. Varani J, Hirschl RB, Dame M, Johnson K 1996 Perfluorocarbon protects lung epithelial cells from neutrophil-mediated injury in an in vitro model of liquid ventilation therapy. Shock 6:339-344 